Pacific Journal of Mathematics

RIGHT CONGRUENCES AND SEMISIMPLICITY FOR RES 


\section{RIGHT CONGRUENCES AND SEMISIMPLICITY FOR REES MATRIX SEMIGROUPS}

\section{ROBERT H. OEHMKe}

In this paper a comparison is made for various definitions of radicals and semisimplicity on the class of Rees matrix semigroups. Preliminary to this, results are obtained on various types of right congruences. This is equivalent to characterizing various types of automata having a Rees matrix semigroup as an input semigroup.

Let $K$ and $L$ be arbitrary sets and $G$ a group. The group $G$ with a zero adjoined is denoted by $G^{0}$. We let $\phi$ be a mapping of $L \times K$ into $G^{0}$ and define a product on $K \times G^{0} \times L$ by

$$
(e, g, f)(u, w, v)=(e, g \phi(f, u) w, v)
$$

If we identify all $(e, 0, f)$ by $\theta$ we obtain a semigroup with zero $\theta$ called a Rees matrix semigroup with zero [1]. If we restrict our attention to $K \times G$ $\times L$ and restrict $\phi$ to be into $G$ then the product in (1) defines a Rees matrix semigroup without zero [1]. These semigroups have played an extremely important role in the characterization of simple semigroups.

Recently, several attempts have been made to obtain a structure theory for semigroups by first defining a radical and, subsequently, semisimplicity. In order to bring the two approaches somewhat closer together we examine the various definitions of semisimplicity as they apply to Rees matrix semigroups. Preliminary to this we characterize modular, maximal modular, and transitive right congruences, as well as the general right congruences, for Rees matrix semigroups.

1. Right congruences of a Rees matrix semigroup. As above, we describe our Rees matrix semigroup with zero using the notation $S=(K \times$ $G \times L) \cup\{\theta\}$ and $(e, 0, f)=\theta$ for all $e \in K$ and $f \in L$. Define $L_{0}=\{f$ : $f \in L$ and $\forall k \in K, \phi(f, k)=0\}, L_{1}=L-L_{0}$ and $f(K)=\{k: k \in K$ and $\phi(f, k) \neq 0\}$. Let

(2) $U$ be a set not containing 1 or 0 and $\left\{K_{i}: i \in U\right\} \cup\left\{K_{0}\right\}$ be a decomposition of $K$ with $K_{0}$ possibly empty. To each $K_{i}$ with $i \in U$ assign a subgroup $H_{i}$ of $G$; to $K_{0}$ assign $G^{0}$.

(3) $\alpha: K \rightarrow G$. 
(4) $L_{i}(a, f)$, for each $i \in U, a \in G$ and $f \in L_{1}$, be a subset of $L_{1}$ such that (a) $f \in L_{i}(a, f)$;

(b) $v \in L_{i}(a, f)$ implies $f(K)=v(K)$; $\left.x)^{-1}, v\right)$

(c) $v \in L_{i}(a, f)$ and $x \in v(K)$ implies $L_{i}(a, f)=L_{i}(a \phi(f, x) \phi(v$,

(d) $a b^{-1} \in H_{i}$ implies $L_{i}(a, f)=L_{i}(b, f)$.

In terms of the above selections we define a relation $\sigma$ on $I=\left(K \times G \times L_{1}\right)$ $\cup\{\theta\}$ as follows:

(5) for $i \in U$ and $e, u \in K_{i}$ we let $(e, g, f) \sigma(u, w, v)$ if and only if for every $k \in f(K)$ we have

$$
\begin{gathered}
\alpha(e)^{-1} g \phi(f, k) \phi(v, k)^{-1} w^{-1} \alpha(u) \in H_{i}, \text { and } \\
v \in L_{i}\left(\alpha(e)^{-1} g, f\right) .
\end{gathered}
$$

(6) All elements of $\left(K_{0} \times G \times L_{1}\right) \cup\{\theta\}$ are $\sigma$-related.

We first show $\sigma$ is an equivalence relation. Since $\sigma$ is defined separately on $\left(K-K_{0}\right) \times G \times L_{1}$ and $\left(K_{0} \times G \times L_{1}\right) \cup\{\theta\}$ by (5) and (6), respectively, and $\sigma$ is trivial on the later subset we need only consider $\sigma$ 's behavior on $\left(K-K_{0}\right) \times G \times L_{1}$. Let $(e, g, f)$ and $(u, w, v)$ be in $\left(K-K_{0}\right)$ $\times G \times L_{1}$. Clearly, $(e, g, f) \sigma(e, g, f)$. If $(e, g, f) \sigma(u, w, v)$ then there exists an $i \neq 0$ such that $e, u \in K_{i}$ and for $k \in v(K)$ we have $\alpha(e)^{-1} g \phi(f, k) \phi(v$, $k)^{-1} w^{-1} \alpha(u) \in H_{i}$. Thus $u, e \in K_{i}$ and

$$
\begin{aligned}
& \alpha(u)^{-1} w \phi(v, k) \phi(f, k)^{-1} g^{-1} \alpha(e)= \\
& {\left[\alpha(e)^{-1} g \phi(f, k) \phi(v, k)^{-1} w^{-1} \alpha(u)\right]^{-1} \in H_{i} .}
\end{aligned}
$$

Also, $v \in L_{i}\left(\alpha(e)^{-1} g, f\right)=L_{i}\left(\alpha(e)^{-1} g \phi(f, x) \phi(v, x)^{-1}, v\right)$ for $x \in f(K)$. But $H_{i} \alpha(e)^{-1} g \phi(f, x) \phi(v, x)^{-1}=H_{i} \alpha(u)^{-1} w$. Hence, $f \in L_{i}\left(\alpha(u)^{-1} w, v\right)$ and $(u$, $w, v) \sigma(e, g, f)$. To prove transitivity assume $(e, g, f) \sigma(u, w, v)$ and $(u, w, v) \sigma(x$, $z, y)$. By the definition of a decomposition there must be an $i \neq 0$ such that $e, u, x \in K_{i}$. Also ${ }_{\alpha}(e)^{-1} g \phi(f, k) \phi(v, k)^{-1} w^{-1} \alpha(u) \in H_{i}$ and

$$
\begin{gathered}
\alpha(u)^{-1} w \phi(v, k) \phi(y, k)^{-1} z^{-1} \alpha(x) \in H_{i} \text { for } \\
k \in v(K), v \in L_{i}\left(\alpha(e)^{-1} g, f\right) \text { and } y \in L_{i}\left(\alpha(u)^{-1} w, v\right) .
\end{gathered}
$$

As above, 
$y \in L_{i}\left(\alpha(u)^{-1} w, v\right)=L_{i}\left(\alpha(u)^{-1} w \phi(v, k) \phi(f, k)^{-1}, f\right)=L_{i}\left(\alpha(e)^{-1} g, f\right)$ and $\alpha(e)^{-1} g \phi(f, k) \phi(y, k)^{-1} z^{-1} \alpha(x)$

$=\left[\alpha(e)^{-1} g \phi(f, k) \phi(v, k)^{-1} w^{-1} \alpha(u)\right]\left[\alpha(u)^{-1} w \phi(v, k) \phi(y, k)^{-1} z^{-1} \alpha(x)\right] \in$ $H_{i}$.

Hence $(e, g, f) \sigma(x, z, y)$ and $\sigma$ is transitive.

We now show $\sigma$ is a right congruence on $I$. Clearly, $\left(K_{0} \times G \times L_{1}\right) \cup$ $\{\theta\}$ is a right ideal of $I$ so again we need only consider right multiplication operating on equivalence classes in $\left(K-K_{0}\right) \times G \times L_{1}$. Thus assume (e, $g, f)$ and $(u, w, v)$ are in $\left(K-K_{0}\right) \times G \times L_{1}$ and are congruent. For $(x, z$, $y) \in I$ let

$$
\begin{aligned}
& a=(e, g, f)(x, z, y)=(e, g \phi(f, x) z, y) \\
& b=(u, w, v)(x, z, y)=(u, w \phi(v, x) z, y) .
\end{aligned}
$$

Since $e, u \in K_{i}$ for some $i \neq 0, g, w \in G$ and $v \in L_{i}\left(\alpha(e)^{-1} g, f\right)$ we see $a$ is in $\left(K_{0} \times G \times L_{1}\right) \cup\{\theta\}$ only if $a=\theta$. But then we would have $\phi(f, x) z=$ 0 ; by (4b), $\phi(v, x) z=0$ and $b=\theta$. Thus $a \sigma b$ in this case. So assume $a$ is in $\left(K-K_{0}\right) \times G \times L_{1}$ (and by symmetry, so is (b). For $k^{\prime} \in y(K)$ we have

$$
\begin{gathered}
\alpha(e)^{-1}(g \phi(f, x) z) \phi\left(y, k^{\prime}\right) \phi\left(y, k^{\prime}\right)^{-1}\left(z^{-1} \phi(v, x)^{-1} w^{-1}\right) \alpha(u) \\
=\alpha(e)^{-1} g \phi(f, x) \phi(v, x)^{-1} w^{-1} \alpha(u) \in H_{i}
\end{gathered}
$$

and again $a \sigma b$. Thus $\sigma$ is a right congruence on $I$.

To extend $\sigma$ to a right congruence on $S$ we let $\bar{\sigma}$ be any equivalence relation on $\left(K \times G \times L_{0}\right) \cup\{\theta\}$ such that

$$
(e, g, f) \sigma(u, w, v) \Rightarrow(e, g \phi(f, k) p, l) \bar{\sigma}(u, w \phi(v, k) p, l)
$$

for any $e, u, k \in K ; p, g, w \in G^{0} ; f, v \in L_{1}$; and $l \in L_{0}$. The extension of $\sigma$ to all of $S$ is then the equivalence relation $\delta$ corresponding to the decomposition

(8) $\left(K_{0} \times G \times L_{1}\right) \cup V_{1}, T_{1}, T_{2}, \ldots, V_{2}, V_{3}$, where $\left(K_{0} \times G \times L_{1}\right) \cup\{\theta\}, T_{1}, \ldots$ are the equivalence classes of $\sigma$ and $V_{1}, V_{2} \ldots$ are the equivalence classes of $\bar{\sigma}$ with $\theta \in V_{1}$.

Then $\delta$ is a right congruence on $S$ by (7) and the fact that the elements of $K$ $\times G \times L_{0}$ are total left divisors of zero.

We have proven half of the following 
THEOREM 1. The set of right congruences on $S$ is characterized as the set of relations $\delta$ defined by (2)-(8).

Proof. It remains to show every right congruence $\delta$ on $S$ can be defined in this manner. So let $\sigma$ be $\delta$ restricted to $I=\left(K \times G \times L_{1}\right) \cup\{\theta\}$ and $\bar{\sigma}$ be $\delta$ restricted to $\left(K \times G \times L_{0}\right) \cup\{\theta\}$. If $(e, 0, f) \sigma(u, w, v) ; \phi(v, k) \neq$ $0 ; w, g \in G$; and $m \in L_{1}$ then the elements $(e, 0, f),(e, 0, f)(k, \phi(v$, $\left.k)^{-1} w^{-1} g, m\right),(u, w, v)\left(k, \phi(v, k)^{-1} w^{-1} g, m\right)$, and $(u, g, m)$ are congruent modulo $\sigma$. Thus if there are any nonzero elements of $I$ in the equivalence class of $\delta$ containing $\theta$ they form a set $\left(K_{0} \times G \times L_{1}\right) \cup\{\theta\}$ and the set of equivalence classes of $\delta$ are as in (8). Since $\delta$ is a right congruence, (7) clearly holds.

If $(e, g, f)$ and $(u, w, v)$ are in $\left(K-K_{0}\right) \times G \times L_{1}$ and $(x, y, z)$ is in $I$, then

$$
(e, g, f) \sigma(u, w, v) \Rightarrow(e, g \phi(f, x) z, y) \sigma(u, w \phi(v, x) z, y)
$$

Define

$$
H(e, f)=\{g: g \in G \text { and }(e, g, f) \sigma(e, 1, f)\}
$$

Let $u=e, v=f=y, \phi(f, x) \neq 0$, and $z=\phi(f, x)^{-1} g^{-1}$ in (9) to obtain

$$
(e, g, f) \sigma(e, w, f) \Rightarrow(e, 1, f) \sigma\left(e, w g^{-1}, f\right)
$$

Thus $H(e, f)$ is a subgroup of $G$. More generally, the intersection of $\{e\} \times$ $G \times\{f\}$ with an equivalence class of $\sigma$ is either empty or a subset $\{e\} \times$ $H(e, f) a \times\{f\}$. Let $u=e, w=1, v=f, \phi(f, x) \neq 0$, and $z=\phi(f, x)^{-1}$ in (9) to obtain

$$
(e, g, f) \sigma(e, 1, f) \Rightarrow(e, g, y) \sigma(e, 1, y)
$$

Thus $H(e, f) \subseteq H(e, y)$ and by symmetry $H(e, f)=H(e, y)=H(e)$. Let $u=$ $e, \phi(f, s) \neq 0$, and $z=\phi(f, x)^{-1} g^{-1}$ in (9) to obtain

$$
(e, g, f) \sigma(e, w, v) \Rightarrow(e, 1, y) \sigma\left(e, w \phi(v, x) \phi(f, x)^{-1} g^{-1}, y\right)
$$

and $w \phi(v, x) \phi(f, x)^{-1} g^{-1} \in H(e)$ and $\phi(v, x) \neq 0$. For each $(e, g, f)$ we let $L(e, g, f)=\left\{v: v \in L_{1}\right.$ and there exists a $w$ with $\left.(e, g, f) \sigma(e, w, v)\right\}$. Clearly, $f \in L(e, g, f)$ and $L(e, g, f)=L(e, w, v)$. Since $w \phi(v, x) \phi(f, x)^{-1} g^{-1} \in H(e)$ 
we have $L(e, g, f)=L\left(e, w \phi(v, x) \phi(f, x)^{-1}, f\right)=L(e, w, v)$. Thus $f \in L(e, w$, $v)$ implies $L\left(e, w \phi(v, x) \phi(f, x)^{-1}, f\right)=L(e, w, v)$.

We define a relation $\rho$ in $K-K_{0}$ by $e \rho u$ if and only if there are $g, w \in G$ and $f, v \in L_{1}$ such that $(e, g, f) \sigma(u, w, v)$. If $(e, g, f) \sigma(u, w, v)$ and $(u, p, q) \sigma(x, z, y)$ we see $(u, w, v) \sigma\left(x, z \phi(y, k) \phi(q, k)^{-1} p^{-1} w, v\right)$ for $\phi(y, k) \neq$ 0 .

Thus $e \rho u$ and $u \rho x$ implies $e \rho x$ and $\rho$ is an equivalence relation on $K-K_{0}$ with related decomposition $\left\{K_{i}: i \in U\right\}$.

Let $\phi(f, x) \neq 0, z=\phi(f, x)^{-1} g^{-1} h g, h \in H(e)$, and $y=f$ in (9) to get

$$
(e, g, f) \sigma(u, w, v) \Rightarrow(e, h g, f) \sigma\left(u, w \phi(v, x) \phi(f, x)^{-1} g^{-1} h g, f\right)
$$

Therefore $(u, w, v) \sigma\left(u, w \phi(v, x) \phi(f, x)^{-1} g^{-1} h g, f\right)$ and by letting $h=1$ we see $f \in L(u, w, v)$. Thus $L(e, g, f) \subseteq L(u, w, v)$ and by symmetry $L(e, g, f)=$ $L(u, w, v)$. We also see that

$$
w \phi(v, x) \phi(f, x)^{-1} g^{-1} h g \phi(f, x) \phi(v, x)^{-1} w^{-1} \in H(u),
$$

i.e.,

$$
w \phi(v, x) \phi(f, x)^{-1} g^{-1} H_{e} g \phi(f, x) \phi(v, x)^{-1} w^{-1} \subseteq H(u) .
$$

By symmetry,

$$
g \phi(f, x) \phi(v, x)^{-1} w^{-1} H_{u} w \phi(v, x) \phi(f, x)^{-1} g^{-1} \subseteq H(e) .
$$

Thus equality holds and the two subgroups are conjugates. For some choice of $e$ in $K_{i}$ we let $H_{i}=H_{e}, \alpha(e)=1$, and

$$
\alpha(u)=w \phi(v, x) \phi(f, x)^{-1} g^{-1}
$$

where $w, v, x$, $f$ and $g$ are as above. Then $\alpha(u) H_{i} \alpha(u)^{-1}=H(u)$ and

$$
g \phi(f, x) \phi(v, x)^{-1} w^{-1} \alpha(u) \in H_{i} .
$$

If $(e, r, s)$ and $(u, p, q)$ is another pair of congruent elements then

$$
(e, g, f) \sigma\left(u, p \phi(q, k) \phi(s, k)^{-1} r^{-1} g, f\right) \sigma(u, w, v)
$$


and

$$
p \phi(q, k) \phi(s, k)^{-1} r^{-1} g \phi(f, k) \phi(v, k)^{-1} w^{-1} \in H(u) .
$$

Thus $p \phi(q, k) \phi(s, k)^{-1} r^{-1} \in \alpha(u) H_{i}$, i.e., $\alpha(u)$ is unique up to a right multiple by an element of $H_{i}$. Now if $(e, g, f) \sigma(u, w, v) \sigma(x, z, y)$ then $g \phi(f$, $k) \phi(v, k)^{-1} w^{-1} \alpha(u)$ and $g \phi(f, k) \phi(y, k)^{-1} z^{-1} \alpha(x)$ are in $H_{i}$ and hence

$$
\alpha(x)^{-1} z \phi(y, k) \phi(v, k)^{-1} w^{-1} \alpha(u) \in H_{i} .
$$

If $p q^{-1} \in H(u)$ then $\left(\alpha(u)^{-1} p\right)\left(\alpha(u)^{-1} q\right)^{-1} \in H_{i}$. Hence the mapping $H(u) p \rightarrow H_{i} \alpha(u)^{-1} p$ is independent of the representative $p$. Thus the assignment of $L(u, w, v)$ to the triple $(u, H(u) w, v)$ can be considered as an assignment to $\left(u, h_{i} \alpha(u)^{-1} w, v\right)$. If $x$ is also in $K_{i}$ there exist $r, z \in G$ and $t, y$ $\in L_{1}$ such that $(u, r, t) \sigma(x, z, y)$. Then if $\phi(t, k) \neq 0$ we have

$$
(u, w, v) \sigma\left(x, z \phi(y, k) \phi(t, k)^{-1} r^{-1} w, v\right)
$$

and

$$
\alpha(x)^{-1} z \phi(y, k) \phi(t, k)^{-1} r^{-1} \alpha(u) \in H_{i}
$$

Therefore

$$
(u, w, v) \sigma\left(x, \alpha(x) h_{i} \alpha(u)^{-1} w, v\right)
$$

and

$$
L(u, w, v)=L\left(x, \alpha(x) h_{i} \alpha(u)^{-1} w, v\right)
$$

Thus the subset of $L_{1}$ assigned to $\left(u, H_{i} \alpha(u)^{-1} w, v\right)$ is the same as the one assigned to $\left(x, H_{i} \alpha(u)^{-1} w, v\right)$ and hence is independent of $u$. We write $L(u$, $w, v)=L_{i}\left(\alpha(u)^{-1} w, v\right)$.

Finally we let $u, x \in K_{i}, y \in L_{i}\left(\alpha(u)^{-1} w, v\right)$, and

$$
\alpha(u)^{-1} w \phi(v, k) \phi(y, k)^{-1} z^{-1} \alpha(x) \in H_{i} .
$$

As we have seen above, if $u, x \in K_{i}$ then

$$
(u, w, v) \sigma\left(x, \alpha(x) h_{i} \sigma(u)^{-1} w, v\right)
$$


for some $h_{i} \in H_{i}$. Since $y \in L_{0}\left(\alpha(u)^{-1} w, v\right)$ there exists an $m \in G$ such that $(u, m, y) \sigma(u, w, v)$ and

$$
\alpha(u)^{-1} m \phi(y, k) \phi(v, k)^{-1} w^{-1} \alpha(u) \in H_{i} .
$$

Then $\alpha(u)^{-1} m z^{-1} \alpha(x) \in H_{i}$ and

$$
\begin{aligned}
(u, m, y) \sigma\left(x, \alpha(x) h_{i} \alpha(u)^{-1} w w^{-1} m, y\right) & =\left(x, \alpha(x) h_{i} \alpha(u)^{-1} m, y\right) \\
= & \left(x, \alpha(x) h_{i} h_{i}^{\prime} \alpha(x)^{-1} z, y\right) \sigma(x, z, y) .
\end{aligned}
$$

Thus $\delta$ is defined as in (2)-(8) and the proof of the theorem is complete.

The situation is slightly less complicated for Rees matrix semigroups without zero. However, we can obtain a characterization of the class of right congruences from the previous characterization by adjoining a zero, $\theta$, to $S$. It is only necessary to characterize those right congruences of $S \cup\{\theta\}$ for which $\{\theta\}$ is an equivalence class. We use Theorem 1 to obtain

THEOREM 2. Let $S$ be a Rees matrix semigroup without zero. The right congruences $\delta$ on $S$ can be characterized as follows: Let

(11) $\left\{K_{i}: i \in U\right\}$ be a decomposition of $K$ such that to each $K_{i}$ is assigned a subgroup $H_{i}$ of $G$;

(12) $\alpha: K \rightarrow G$;

(13) for each $H_{i}$ a and $f \in L$ let $L_{i}(a, f)$ be a subset of $L$ such that

(a) $f \in L_{i}(a, f)$,

(b) $v \in L_{i}(a, f) \Rightarrow L_{i}(a, f)=L_{i}\left(a \phi(f, x) \phi(v, x)^{-1}, v\right)$;

(14) $(e, g, f) \delta(u, w, v) \Longleftrightarrow \exists$ i such that $e, u \in K_{i}, v \in L_{i}\left(\alpha(e)^{-1} g, f\right)$ and for every $k \in K$,

$$
\alpha(e)^{-1} g \phi(f, k) \phi(v, k)^{-1} w^{-1} \alpha(u) \in H_{i}
$$

2. Modularity, transitivity and maximality. We shall first examine which right congruences are modular, i.e., for which right congruences $\delta$ does an element $(e, g, f)$, called a left identity for $\delta$, exist such that for all $(u$, $w, v)$ in $\delta$

$$
(e, g, f)(u, w, v) \delta(u, w, v)
$$

Of course the universal relation $v$ is a modular right congruence with each element a left identity. We concern ourselves with right congruences $\delta \neq v$. 
THEOREM 3. A necessary and sufficient condition that the right congruence $\delta \neq v$ is modular is (a) $K=K_{0} \cup K_{1}$, (b) there exists an $f \in L$ such that for $e, u \in K_{1}$ and $k \in K_{0}$ we have $\phi(f, e) \neq 0, \phi(f, k)=0$, and $\alpha(e)^{-1} \phi(f, e)^{-1} \phi(f, u) \alpha(u) \in H_{1}$. The element $\left(e, \phi(f, e)^{-1}, f\right)$ will then be a left identity for $\delta$.

Proof. If $\delta \neq v$ is modular with left identity $(e, g, f)$ then $(e, g, f)$ is not contained in any equivalence class that is a right ideal. For then we would have $\delta=v$. Therefore $(e, g, f) \notin\left(K_{0} \times G \times L_{1}\right) \cup\left(K \times G \times L_{0}\right)$ $\cup\{\theta\}$. Hence $e \in K-K_{0}, g \in G$, and $f \in L_{1}$. From (15) we see $(e, g \phi(f$, $e) g, f) \delta(e, g, f)$. Therefore $\phi(f, e) \neq 0$ and $g \in H_{e} \phi(f, e)^{-1}$. Since any element congruent to a left identity is also a left identity we have $(e, \phi(f$, $\left.e)^{-1}, f\right)$ a left identity. If $(u, w, v)$ is in $\left(K-K_{0}\right) \times G \times L_{1}$ then by (14)

$$
\left(e, \phi(f, e)^{-1} \phi(f, u) w, v\right) \sigma(u, w, v)
$$

Therefore $e$ and $u$ are in the same decomposition class of $K$ and hence $K=$ $K_{0} \cup K_{1}$. Also $\phi(f, u) \neq 0$ and

$$
\alpha(e)^{-1} \phi(f, e)^{-1} \phi(f, u) \alpha(u) \in H_{i} .
$$

If $(u, w, v) \in K_{0} \times G \times L_{1}$ then

$$
\left(e, \phi(f, e)^{-1} \phi(f, u) w, v\right) \in\left(K_{0} \times G \times L_{1}\right) \cup\{\theta\}
$$

Since $e \notin K_{0}$ we have $\phi(f, e)^{-1} \phi(f, u) w=0$ and $\phi(f, u)=0$. Therefore (a) and (b) are satisfied.

Conversely, if (a) and (b) are satisfied consider

$$
\left(e, \phi(f, e)^{-1}, f\right)(u, w, v)=\left(e, \phi(f, e)^{-1} \phi(f, u) w, v\right) .
$$

If $(u, w, v) \in K_{1} \times G \times L_{1}$ then $\alpha(e)^{-1}\left(\phi(f, e)^{-1} \phi(f, u) w\right) w^{-1} \alpha(u) \in H_{i}$ by (b) and

$$
\left(e, \phi(f, e)^{-1} \phi(f, u) w, v\right) \delta(u, w, v)
$$

If $(u, w, v) \in K_{0} \times G \times L_{1}$ then $\phi(f, u)=0$ and again

$$
\left(e, \phi(f, e)^{-1} \phi(f, u) w, v\right) \delta(u, w, v)
$$

If $(u, w, v) \in K \times G \times L_{0}$ then $(u, w, f) \in K \times G \times L_{1}$ so 


$$
(u, w, f) \delta\left(e, \phi(f, x)^{-1} \phi(f, u) w, f\right)
$$

By multiplying on the right by $\left(e, \phi(f, e)^{-1}, v\right)$ we have

$$
(u, w, v) \delta\left(e, \phi(f, e)^{-1}, f\right)(u, w, v) \text {. }
$$

Clearly, $\left(e, \phi(f, e)^{-1}, f\right) \theta \delta \theta$ and hence $\delta$ is modular.

A right congruence $\delta$ is transitive if for every $(e, g, f)$ and $(u, w, v)$ in $S$ there is an $(x, z, y)$ such that $(e, g, f)(x, z, y) \delta(u, w, v)$. If $S$ contains a zero element the equivalence class containing zero is a right ideal. Hence $\delta$ cannot be transitive unless $\delta=v$.

For $S$ containing a zero element $\theta$ we say $\delta$ is $\theta$-transitive if for every (e, $g, f) \not \equiv \theta(\bmod \delta)$ and $(u, w, v)$ in $S$ there is an $(x, y, z)$ in $S$ such that $(e, g$, $f)(x, z, y) \delta(u, w, v)$.

THEOREM 4. Let $S$ be without a zero element. Then a right congruence $\delta$ is transitive if and only if $K=K_{1}$.

Proof. Assume $\delta$ is a right congruence such that $K=K_{1}$. Let $(e, g, f)$ and $(u, w, v)$ be elements of $S$. Then

$$
(e, g, f)\left(u, \phi(f, u)^{-1} g^{-1} \alpha(e) \alpha(u)^{-1} w, v\right)=\left(e, \alpha(e) \alpha(u)^{-1} w, v\right) .
$$

Since $e, u \in K_{1}$ and

$$
\alpha(e)^{-1}\left(\alpha(e) \alpha(u)^{-1} w\right) w^{-1} \alpha(u) \in H_{i}
$$

we have

$$
\left(e, \alpha(e) \alpha(u)^{-1} w, v\right) \delta(u, w, v)
$$

and $\delta$ is transitive.

Conversely, if $\delta$ is transitive and $(e, g, f)(x, z, y) \delta(u, w, v)$ then $e$ and $u$ are in the same decomposition class of $K$; hence $K=K_{1}$.

THEOREM 5. Let $S$ be a Rees matrix semigroup with a zero $\theta$. Then $\delta, a$ right congruence on $S$, is $\theta$-transitive if and only if (a) $K=K_{0} \cup K_{1}$ and (b) $\left(K_{0} \times G \times L_{1}\right) \cup\left(K \times G \times L_{0}\right) \cup\{\theta\}$ forms a single equivalence class.

Proof. Assume $\delta$ is $\theta$-transitive and $(e, g, f) \neq \equiv(\bmod \delta)$. Then there exists an $(x, z, y)$ such that $(e, g, f)(x, z, y) \delta(e, g, f)$. But if $f \in L_{0}$ we have 
$\phi(f, x)=0$ and $\theta \delta(e, g, f)$. Thus all the elements of $K \times G \times L_{0}$ are congruent to $\theta$ and condition (b) holds. So assume $(e, g, f)$ and $(u, w, v)$ are in $\left(K-K_{0}\right) \times G \times L_{1}$. Since there is an $(x, z, y)$ such that $(e, g, f)(x, z$, $y) \delta(u, w, v)$ we have $e$ and $u$ in the same decomposition class of $K-K_{0}$; i.e., $K-K_{0}=K_{1}$.

Conversely, if (a) and (b) hold, $(e, g, f) \neq \theta(\bmod \delta)$ and $(u, w, v) \in S$ with $u \in K-K_{0}$ and $w \neq 0$ choose $x \in K$ such that $\phi(f, x) \neq 0$. We can readily see that

$$
(e, g, f)\left(x, \phi(f, x)^{-1} g^{-1} \alpha(e) \alpha(u)^{-1} w, v\right) \delta(u, w, v) .
$$

If $(u, w, v) \in\left(K_{0} \times G \times L_{1}\right) \cup\left(K \times G \times L_{0}\right) \cup\{\theta\}$ then

$(e, g, f)(e, 0, f) \delta \theta \delta(u, w, v)$

Hence $\delta$ is $\theta$-transitive.

For semigroups without a zero element a definition of zero-transitivity for right congruences is still possible. If $U$ is an equivalence class of the right congruence $\delta$, that is, a right ideal, then $\delta$ is said to be $U$-transitive if for every $a \notin U$ and $b \in S$ there is a $c \in S$ such that $a c \delta b$. However, in a Rees matrix semigroup without zero, for such a right congruence $\delta$ we can show in much the same way as above that the decomposition of $K$ associated to $\delta$ consists of a single subset, namely, $K$. But if $(e, g, f) \in U$ then $e$ $\times G \times L \subseteq U$ and $\delta=v$.

The set of all right congruences on a semigroup $S$ can be partially ordered in the same way equivalence relations are ordered, namely, $\delta \leq \bar{\delta}$ if and only if ( $a, b \in S, a \delta b \Rightarrow a \bar{\delta} b$ ).

THEOREM 6. Let $\delta$ and $\bar{\delta}$ be two right congruences on a Rees matrix semigroup $S$ defined by $K_{0}, \ldots, \alpha, H_{1}, \ldots, L_{1}(a, f), \ldots$, etc., and $\bar{K}_{0}, \ldots, \bar{\alpha}$, $\bar{H}_{1}, \ldots, \bar{L}_{1}(a, f), \ldots$, etc., respectively. Then $\delta \leq \delta$ if and only if

(a) $K_{0} \subseteq \bar{K}_{0}$,

(b) $\forall i \neq 0 \exists q$ such that $K_{i} \subseteq \bar{K}_{q}$,

(c) for $q, i \neq 0$ and $e, u \in K_{i}$ we have $H_{i} \subseteq \alpha(e)^{-1} \bar{\alpha}(e) \bar{H} q \bar{\alpha}(u)^{-1} \alpha(u)$,

(d) for every $a \in G$ and $f \in L_{1}$ we have $L_{i}(a, f) \subseteq \bar{L} q(a, f)$,

(e) $\delta$ restricted to $\left(K \times G \times L_{0}\right) \cup\{\theta\}$ is less than or equal to $\delta$ restricted to $\left(K \times G \times L_{0}\right) \cup\{\theta\}$.

Proof. Assume (a)-(e) hold. We need only show the restrictions of $\delta$ and $\delta$ to $\left(K \times G \times L_{1}\right) \cup\{\theta\}$ satisfy the proper inequality. Assume $(e, g$, $f) \delta(u, w, v)$. If $(e, g, f),(u, w, v) \in\left(K_{0} \times G \times L_{1}\right) \cup\{\theta\}$ it is clear that $(e, g$, 
$f) \delta(u, w, v)$ from $K_{0} \subseteq \bar{K}_{0}$. So assume $e, u \in K_{i}, w g \neq 0, q \neq 0$, and $e, u \in$ $\bar{K}_{q}$. Then

$$
\begin{aligned}
\alpha(e)^{-1} g \phi(f, x) \phi(v, x)^{-1} w^{-1} \alpha(u) \in \\
H_{i} \subseteq \alpha(e)^{-1} \bar{\alpha}(e) \bar{H}_{q} \bar{\alpha}(u)^{-1} \alpha(u) .
\end{aligned}
$$

Therefore $\bar{\alpha}(e)^{-1} g \phi(f, x) \phi(v, x)^{-1} w^{-1} \bar{\alpha}(u) \in \bar{H}_{q}$. Also

$$
v \in L_{i}\left(\alpha(e)^{-1} g, f\right) \subseteq \bar{L}_{q}\left(\bar{\alpha}(e)^{-1} g, f\right)
$$

Hence $(e, g, f) \delta(u, w, v)$ and $\delta \leq \delta$.

Conversely, assume $\delta \leq \bar{\delta}$ and $e \in K_{0}$. For every $g \in G$ and $f \in L$, we have $(e, g, f) \delta \theta$ and therefore $(e, g, f) \delta \theta$. Thus $e \in \bar{K}_{0}$ and $K_{0} \subseteq \bar{K}_{0}$. If $e, u \in$ $K_{i}$ for $i \neq 0$ there exist $g, w \in G$ and $f, v \in L$, such that $(e, g, f) \delta(u, w, v)$. Then $(e, g, f) \bar{\delta}(u, w, v)$ and there must exist a $q$ such that $e, u \in \bar{K}_{q}$. Hence $K_{i} \subseteq \bar{K}_{q}$. For $h \in H_{i}, q \neq 0$, and $v \in L_{i}(1, f)$ we have

$$
\left(e, \alpha(e) h \phi(f, x)^{-1}, f\right) \delta\left(u, \alpha(u) \phi(v, x)^{-1}, v\right)
$$

and hence

$$
\left(e, \alpha(e) h \phi(f, x)^{-1}, f\right) \delta\left(u, \alpha(u) \phi(v, x)^{-1}, v\right)
$$

Therefore,

$$
\begin{gathered}
\bar{\alpha}(e)^{-1} \alpha(e) h \alpha(u)^{-1} \bar{\alpha}(u) \in \bar{H}_{q} \text { and } \\
H_{i} \subseteq \alpha(e)^{-1} \bar{\alpha}(e) \bar{H}_{q} \bar{\alpha}(u)^{-1} \alpha(u) .
\end{gathered}
$$

If $e, u \in K_{i}, i \neq 0, a \in G$, and $v \in L_{i}(a, f)$ then $(e, \alpha(e) a, f) \delta$

$$
\left(u, \alpha(u) a \phi(v, x)^{-1} \phi(f, x), v\right)
$$

and $(e, \alpha(e) a, f) \delta\left(u, \alpha(u) a \phi(v, x)^{-1} \phi(f, x), v\right)$. Therefore, $v \in \bar{L}_{q}(a, f)$ and $L_{i}(a, f) \subseteq \bar{L}_{q}(a, f)$. The proof is complete.

THEOREM 7. Let $S$ be a Rees matrix semigroup without zero and $\delta$ a modular right congruence on $S$ with associated subgroup $H$. If $\bar{H}$ is a subgroup containing $H$ then there is a right congruence $\delta \geq \delta$ with associated subgroup $\bar{H}$.

Proof. If $L(a, f)$ 's are the subset of $L$ defined by $\delta$ let $\bar{L}(a, f)$ be the set 
of all $v \in L$ such that there are sequences $f=v_{0}, v_{1}, \ldots, v_{n}=v$ in $L$ and $b_{1}$, $\ldots, b_{n}$ in $G$ such that $a b_{i}^{-1} \in \bar{H}$ and

$$
v_{i} \in L\left(b_{i} \phi(f, x) \phi\left(v_{i-1}, x\right)^{-1}, v_{i-1}\right)
$$

As we have already seen, this definition is independent of $x$. If we let $y_{i}=$ $v_{n-i}$ and

$$
c_{i}=b_{n+1-i} \phi(f, x) \phi(v, x)^{-1}
$$

we have $v=y_{0}, y_{1}, \ldots, y_{n}=f$ a sequence in $L$ and $c_{1}, \ldots, c_{n}$ a sequence in $G$ such that

$$
\left(a \phi(f, x) \phi(v, x)^{-1}\right) c_{i}^{-1}=a b_{n+1-i}^{-1} € \bar{H}
$$

and

$$
\begin{array}{r}
y_{i-1}=v_{n-i+1} \in L\left(b_{n+1-i} \phi(f, x) \phi\left(v_{n-i}, x\right)^{-1}, v_{n-i}\right)= \\
L\left(c_{i} \phi(v, x) \phi\left(y_{i}, x\right)^{-1}, y_{i}\right) .
\end{array}
$$

The latter implies $y_{i} \in L\left(c_{i} \phi(v, x) \phi\left(y_{i-1}, x\right)^{-1}, y_{i-1}\right)$ and

$$
f \in \bar{L}\left(a \phi(f, x) \phi(v, x)^{-1}, v\right)
$$

Now let $v \in \bar{L}(a, f)$ and $w \in \bar{L}\left(a \phi(f, x) \phi(v, x)^{-1}, v\right)$. Then there are sequences $f=v_{0}, v_{1}, \ldots, v_{n}=v$ and $v=w_{0}, w_{1}, \ldots, w_{m}=w$ in $L$ and sequences $b_{1}, \ldots, b_{n}$ and $c_{1}, \ldots, c_{m}$ in $G$ such that $a b_{i}^{-1} \in \bar{H}$,

$$
\begin{gathered}
a \phi(f, x) \phi(v, x)^{-1} c_{i}^{-1} \in \bar{H}, \\
v_{i} \in L\left(b_{i} \phi(f, x) \phi\left(v_{i-1}, x\right)^{-1}, v_{i-1}\right)
\end{gathered}
$$

and

$$
w_{i} \in L\left(c_{i} \phi(v, x) \phi\left(w_{i-1}, x\right)^{-1}, w_{i-1}\right) .
$$

If we let $w_{i}=v_{n+i}$ and

$$
c_{i}=b_{n+i} \phi(f, x) \phi(y, x)^{-1}
$$

we have $f=v_{0}, \ldots, v_{n+m}=w$ a sequence in $L$ and $b_{1}, \ldots, b_{n+m}$ a sequence in $G$ such that $a b_{i}^{-1} \in \bar{H}_{i}$ and 


$$
v_{i} \in L\left(b_{i} \phi(f, x) \phi\left(v_{i-1}, x\right)^{-1}, v_{i-1}\right)
$$

Therefore, $w \in \bar{L}(a, f)$ and

$$
\bar{L}\left(a \phi(f, v) \phi(v, x)^{-1}, v\right) \subseteq \bar{L}(a, f) .
$$

Since $f \in \bar{L}\left(a \phi(f, x) \phi(v, x)^{-1}, v\right)$ we have

$$
\begin{gathered}
\bar{L}(a, f) \subseteq \bar{L}\left(a \phi(f, x) \phi(v, x)^{-1} \phi(v, x) \phi(f, x)^{-1}, f\right) \\
\subseteq \bar{L}\left(a \phi(f, x) \phi(v, x)^{-1}, v\right) .
\end{gathered}
$$

Hence $\bar{L}(a, f)=\bar{L}\left(a \phi(f, v) \phi(v, x)^{-1}, v\right)$. We can use $\bar{H}$ and the subsets $\bar{L}(a$, $f$ ) and $\bar{\alpha}=\alpha$ to define a right congruence $\delta$. Clearly $\delta \leq \delta$.

COROLLARY. If $\delta$ is a maximal modular right congruence with associated subgroup $H$ then $H=G$ or $H$ is maximal.

Those maximal modular right congruences $\delta$ having $G$ as the associated subgroup are very easy to obtain and characterize. In the first place they only exist if $L$ contains more than one element. In such a case, if $L=$ $L^{\prime} \cup L^{\prime \prime}$ is a nontrivial decomposition of $L$ (i.e., neither $L^{\prime}$ nor $L^{\prime \prime}$ is empty) then the decomposition $\left(K \times G \times L^{\prime}\right) \cup\left(K \times G \times L^{\prime \prime}\right)$ of $S$ has a related equivalence relation that is a maximal modular right congruence and conversely.

The characterization of the maximal modular right congruences associated with a proper subgroup of $G$ is somewhat more difficult.

LEMMA 1. Let $S$ be a Rees matrix semigroup without zero. Let $H$ be a subgroup of $G$ and $\alpha: K \rightarrow G$. Assume

$$
V=\left\{v: v \in L \text { and } a e, u \in K, \alpha(e)^{-1} \phi(v, e)^{-1} \phi(v, u) \alpha(u) \in H\right\}
$$

is not empty. Let

$$
U=U\left\{u \times \alpha(u) H \alpha(u)^{-1} \phi(v, u)^{-1} \times v: u \in K \text { and } v \in V\right\}
$$

Then there exists a right congruence maximal with respect to the property of having $U$ as an equivalence class of left identities.

Proof. For any $a \in S$ define $T_{a}=\left\{b: b \in S^{1}\right.$ and $\left.a b \in U\right\}$. Define a relation $\delta$ on $S$ by $a \delta b$ if and only if $T_{a}=T_{b}$. As is well-known, $\delta$ is a right 
congruence. If $a \in U$ then $1 \in T_{a}$. Hence $a \delta b$ implies $b \in U$. Thus $U$ is the union of equivalence classes of $\delta$. However, if

$$
\begin{gathered}
a=\left(u, \alpha(u) h \alpha(u)^{-1} \phi(v, u)^{-1}, v\right) \in U \text { and } \\
a(x, z, y)=\left(u, \alpha(u) h \alpha(u)^{-1} \phi(v, u)^{-1} \phi(v, x) z, y\right) \in U
\end{gathered}
$$

we must have $y \in V$ and

$$
\alpha(u) h \alpha(u)^{-1} \phi(v, u)^{-1} \phi(v, x) z=\alpha(u) h^{\prime} \alpha(u)^{-1} \phi(y, u)^{-1}
$$

for some $h^{\prime} \in H$. Since $\alpha(u)^{-1} \phi(v, u)^{-1} \phi(v, x) \alpha(x) \in H$ we have

$$
\begin{gathered}
z \in \alpha(x) H \alpha(u)^{-1} \phi(y, u)^{-1}= \\
\alpha(x) H\left[\alpha(u)^{-1} \phi(y, u)^{-1} \phi(y, x) \alpha(x)\right] \alpha(x)^{-1} \phi(y, x)^{-1}= \\
\alpha(x) H \alpha(x)^{-1} \phi(y, x)^{-1} .
\end{gathered}
$$

Hence $(x, z, y) \in U$. A straightforward computation shows that $U$ is also a subsemigroup. Thus if $a, b \in U$ then $T_{a}=T_{b}=U \cup\{1\}$ and $a \delta b$. Therefore $U$ forms a single equivalence class of $\delta$. By Theorem 3 the elements of $U$ are left identities for $\delta$. If $\delta \leq \delta$ and $U$ is an equivalence class of $\delta$ and $c \delta d$ then $c a \in U$ if and only if $d a \in U$ for any $a \in S$. Thus in the above notation $T_{a}=T_{b}$ and $a \delta b$. Hence $\delta=\delta$ and $\delta$ is maximal with respect to having $U$ as an equivalence class.

LEMMA 2. Assume the $H$ of Lemma 1 is maximal and $\delta \nRightarrow \delta$. Then the subgroup associated to $\delta$ is $G$.

Proof. By Lemma $1 U$ must be properly contained in an equivalence class $\bar{U}$ of $\delta$. Clearly $\bar{U}$ is a set of left identities for $\delta$. Let the subgroup associated to $\delta$ be $\bar{H}$. Then for any $(x, z, y) \in \bar{U}$ we have

$$
\bar{\alpha}(e)^{-1} \phi(y, e)^{-1} \phi(y, k) \bar{\alpha}(k) \in \bar{H} .
$$

Now $H \subseteq \alpha(e)^{-1} \bar{\alpha}(e) \bar{H} \bar{\alpha}(u)^{-1} \alpha(u)=W$ for any $e$ and $u$ in $K$. More particularly,

$$
H \subseteq \alpha(e)^{-1} \bar{\alpha}(e) \bar{H} \bar{\alpha}(e)^{-1} \alpha(e)
$$

Since $H$ is maximal we have

$$
\alpha(e)^{-1} \bar{\alpha}(e) \bar{H} \bar{\alpha}(e)^{-1} \alpha(e)=G
$$


or $H$. If the first possibility holds then $\bar{H}=G$, we are finished; so assume the above conjugate of $\bar{H}$ is equal to $H$. Then

$$
H \subseteq \alpha(e)^{-1} \bar{\alpha}(e) \bar{H} \bar{\alpha}(u)^{-1} \alpha(u) \subseteq \alpha(e)^{-1} \bar{\alpha}(e) \bar{\alpha}(u)^{-1} \alpha(u) H=H .
$$

Hence $H=\alpha(e)^{-1} \bar{\alpha}(e) \bar{H} \bar{\alpha}(u)^{-1} \alpha(u)$ for all $e, u \in K$. But then

$$
\bar{\alpha}(e)^{-1} \phi(y, e)^{-1} \phi(y, k) \bar{\alpha}(k) \in \bar{H}
$$

implies $\alpha(e)^{-1} \phi(y, e)^{-1} \phi(y, k) \alpha(k) \in H$ and $y \in V$. Since

$$
(x, z \phi(y, x), y)=(x, z, y)(x, 1, y) \delta(x, 1, y)
$$

we have $\bar{\alpha}(x)^{-1} z \phi(y, x) \bar{\alpha}(x) \in \bar{H}$; i.e.,

$$
z \in \bar{\alpha}(x) \bar{H} \bar{\alpha}(x)^{-1} \phi(y, x)^{-1}=\alpha(x) H \alpha(x)^{-1} \phi(y, x)^{-1} .
$$

Hence $(x, z, y) \in U$ and we have the contradiction $U=\bar{U}$.

THEOREM 8. Let H be maximal, $\alpha: K \rightarrow G$ and $V$, as defined in Lemma 1 , be nonempty. The right congruence $\delta$ defined in Lemma 1 is maximal if and only if for every $f$ and $v$ in $L$ there are sequences $a_{1}, \ldots, a_{n-1}$ in $G$ and $f=v_{1}$, $\ldots, v_{n}=v$ in $L$ such that for every $x$ and $u$ in $K$ we have

$$
a_{i} \phi\left(v_{i}, x\right) \phi\left(v_{i-1}, x\right)^{-1} \phi\left(v_{i-1}, u\right) \phi\left(v_{i}, u\right)^{-1} a_{i}^{-1} \in H .
$$

We will denote the set of all such maximal subgroups $H$ by $\mathscr{M}$.

Proof. Consider the possibility that

$$
\left(e, \alpha(e)^{-1} a_{i} \phi\left(v_{i}, x\right) \phi\left(v_{i-1}, x\right)^{-1}, v_{i-1}\right)(u, p, q) \in U
$$

and

$$
a_{i} \phi\left(v_{i}, x\right) \phi\left(v_{i-1}, x\right)^{-1} \phi\left(v_{i-1}, u\right) \phi\left(v_{i}, u\right)^{-1} a_{i}^{-1} \in H
$$

Then $q \in V$,

$$
\begin{gathered}
\alpha(e)^{-1} a_{i} \phi\left(v_{i}, x\right) \phi\left(v_{i-1}, x\right)^{-1} \phi\left(v_{i-1}, u\right) p \in \\
\alpha(e)^{-1} H \alpha(e) \phi(q, e)^{-1}
\end{gathered}
$$

and

$$
\alpha(e)^{-1} a_{i} \phi\left(v_{i}, u\right) p \in \alpha(e)^{-1} H \alpha(e) \phi(q, e)^{-1} \text {. }
$$


Hence $\left(e, \alpha(e)^{-1} a_{i}, v_{i}\right)(u, p, q) \in U$. It can be similarly shown that if

$$
\left(e, \alpha(e)^{-1} a_{i}, v_{i}\right)(u, p, q) \in U
$$

then so is

$$
\left(e, \alpha(e)^{-1} a_{i} \phi\left(v_{i}, x\right) \phi\left(v_{i-1}, x\right)^{-1}, v_{i-1}\right)(u, p, q) \in U
$$

Thus by the definition of $\delta$ we have

$$
\left(e, \alpha(e)^{-1} a_{i} \phi\left(v_{i}, x\right) \phi\left(v_{i-1}, x\right)^{-1}, v_{i-1}\right) \delta\left(e, \alpha(e)^{-1} a_{i}, v_{i}\right)
$$

and

$$
a_{i} \phi\left(v_{i}, x\right) \phi\left(v_{i-1}, x\right)^{-1} \phi\left(v_{i-1}, u\right) \phi\left(v_{i}, u\right)^{-1} a_{i}^{-1} \in H
$$

implies $v_{i-1} \in L\left(a_{i}, v_{i}\right)$. Now if $\delta \supsetneqq \delta$ and $H$ is maximal we know $\delta$ must be associated to $G$ by Lemma 2 . Since $G$ has only one coset we must have $L(a$, $f) \subseteq \bar{L}(1, f)$ for every $a$ in $G$ and every $f$. Thus if $v_{i-1} \in L\left(a_{i}, v_{i}\right)$ then $v_{i-1} \in$ $\bar{L}\left(1, v_{i}\right)$. By $4(c)$, we then have $\bar{L}\left(1, v_{i}\right)=\bar{L}\left(1, v_{i-1}\right)$. Therefore if the restrictions of the theorem hold for $H$ we must have $\bar{L}(1, f)=\bar{L}(1, v)$ for all $f$ and $v$ in $L$ and $\delta$ has only the single equivalence class $K \times G \times L$. Thus $\delta$ is maximal.

Conversely, assume $\delta$ is maximal. Let $G=\bar{H}$ and construct the right congruence $\delta$ of Theorem 7 . Since $\delta$ properly contains $\delta$ we must have $\delta=$ $v$ and $\bar{L}(1, f)=L$ for all $f$; i.e., for every $f$ and $v$ in $L$ there are sequences $b_{1}$, $\ldots, b_{n-1}$ in $G$ and $f=v_{0}, v_{1}, \ldots, v_{n}=v$ in $L$ such that

$$
\left.v_{i} \in L\left(b_{i} \phi(f, x) \phi V_{i-1}, x\right)^{-1}, v_{i-1}\right)
$$

But then

$$
\begin{gathered}
\left(x, \alpha(x)^{-1} b_{i} \phi(f, x) \phi\left(v_{i-1}, x\right)^{-1}, v_{i-1}\right) \delta \\
\left(x, \alpha(x)^{-1} b_{i} \phi(f, x) \phi\left(v_{i-1}, x\right)^{-1} \phi\left(v_{i-1}, u\right) \phi\left(v_{i}, u\right)^{-1}, v_{i}\right)
\end{gathered}
$$

and

$$
b_{i} \phi(f, x) \phi\left(v_{i-1}, x\right)^{-1} \phi\left(v_{i}, u\right) \phi\left(v_{i-1}, u\right)^{-1} \phi\left(v_{i-1}, x\right) \phi(f, x)^{-1} b_{i}^{-1} \in H
$$

If we let $a_{i}=b_{i} \phi(f, x) \phi\left(v_{i}, x\right)^{-1}$ we have

$$
a_{i} \phi\left(v_{i}, x\right) \phi\left(v_{i-1}, x\right)^{-1} \phi\left(v_{i}, u\right) \phi\left(v_{i-1}, u\right)^{-1} a_{i}^{-1} \in H
$$


for every $u \in K$ and hence

$$
a_{i} \phi\left(v_{i}, e\right) \phi\left(v_{i-1}, e\right)^{-1} \phi\left(v_{i-1}, u\right) \phi\left(v_{i}, u\right)^{-1} a_{i}^{-1} \in H
$$

for every $e$ and $u$ in $K$. Thus (16) holds.

Because of the presence of a zero element $\theta$ the maximal modular right congruences for a Rees matrix semigroup with zero are considerably easier to obtain.

THEOREM 9. Let $\delta$ be a modular right congruence relation on the Rees matrix semigroup $S$ with zero $\theta$. Let the related decomposition of $K$ be $K=$ $K_{0} \cup K_{1}$ and the decomposition of $L$ related to the equivalence relation $\left\{\left(l, l^{\prime}\right)\right.$ $: l, l^{\prime} \in L$ and $\left.l(K)=l^{\prime}(K)\right\}$ be $L(f), L(v), \ldots$ where $f \in L(f)$. Then $\delta$ is contained in a unique maximal right congruence $\bar{\delta}$ whose equivalence classes are

$$
\begin{gathered}
\left(K_{0} \times G \times L_{1}\right) \cup\left(K \times G \times L_{0}\right) \cup\{\theta\}, K_{1} \times G \times L(f), \\
K_{1} \times G \times L(v), \ldots .
\end{gathered}
$$

Proof. Clearly the conditions (2)-(7) and the conditions of Theorem 6 are satisfied. Thus $\delta$ is a right congruence containing $\delta$. If $\delta$ were not maximal, some of the $L(f)$ 's would have to coalesce, which would be a contradiction of $4(b)$. Therefore $\delta$ is maximal. If $\rho$ is any right congruence containing $\delta$ then $\rho$ must be related to the decomposition $K=K_{0} \cup K_{1}$ determined by $\delta$. Since $\delta$ is completely determined by this decomposition we have $\rho \leq \delta$ and $\delta$ is unique. The existence of maximal modular right congruences then depends only on the existence of the proper type of decomposition of $K$.

3. Semisimplicity for Rees matrix semigroups. Various definitions of radicals for semigroups and semisimplicity have been given in the litirature. We shall investigate some of these as they apply to Rees matrix semigroups.

As one of the possible analogs to the Jacobson radical in ring theory we can define a radical of a semigroup to be the equivalence relation which is the intersection of the maximal modular right congruences of $S[3,5]$. With this definition the semigroup is said to be semisimple in case the radical is the identity relation.

THEOREM 10. A Rees matrix semigroup $S$ without zero is semisimple if 
and only if the intersection of the subgroups in $\mathscr{M}$ is the identity and $\phi(v$, $e)^{-1} \phi(v, u)$ is independent of $v$ only if $e=u$.

Proof. If $S$ is semisimple then for every pair of distinct elements in $S$ there must be a maximal modular right congruence $\delta$ such that these elements are incongruent with respect to $\delta$. In particular, $\left(e, \phi(v, e)^{-1}, v\right)$ and $\left(u, \phi(v, u)^{-1}, \mathrm{v}\right)$ must be separated by some $\delta$ if $e \neq u$ and for any $v$. Let $\delta$ be related to $H$ and $\alpha$. Since $\delta$ is modular there is an $f \in L$ such that $\alpha(k)^{-1} \phi(f, k)^{-1} \phi\left(f, k^{\prime}\right) \alpha\left(k^{\prime}\right) \in H$. In particular, $\alpha(e)^{-1} \phi(f, e)^{-1} \phi(f, u) \alpha(u)$ $\in H$. But $\delta$ separates $\left(e, \phi(v, e)^{-1}, v\right)$ and $\left(u, \phi(v, u)^{-1}, v\right)$. Therefore

$$
\alpha(e)^{-1} \phi(v, e)^{-1} \phi(v, u) \alpha(u) \notin H .
$$

Now if $\phi(v, e)^{-1} \phi(v, u)$ is independent of $v$ we have a contradiction. Since the two elements cannot be separated by a $\delta$ associated to $G$ we must have $\phi(v, e)^{-1} \phi(v, u)$ independent of $v$ implies $e=u$.

There also must be a maximal modular right congruence that separates $(e, g, f)$ and $(e, p, f)$ when $g \neq p$. Since this cannot be accomplished by a $\delta$ associated to $G$ there must be an $H$ in $\mathscr{H}$ such that $\alpha(e)^{-1} g p^{-1} \alpha(e) \notin H$ and $g p^{-1} \notin \alpha(e) H \alpha(e)^{-1}$. But $H \in \mathscr{H}$ implies $\alpha(e) H \alpha(e)^{-1} \in \mathscr{M}$. Therefore the intersection of all the subgroups in $\mathscr{M}$ must be $\{1\}$.

Conversely, if the conditions of the theorem hold then any pair $(e, g$, $f)$ and $(u, w, v)$ can be separated if $f \neq v$. If $f=v$ and $e \neq u$ there is an $l \in$ $L$ such that $\phi(l, e)^{-1} \phi(l, u) \neq g w^{-1}$. Let $\alpha(k)=\phi(l, k)^{-1}$ for all $k \in K$ and $H$ an element of $\mathscr{M}$ such that $\phi(l, e) g w^{-1} \phi(l, u)^{-1}$ is not in $H$. We have

$$
\alpha(k)^{-1} \phi(l, k)^{-1} \phi\left(l, k^{\prime}\right) \alpha\left(k^{\prime}\right)
$$

in $H$ for all $k$ and $k^{\prime}$ in $K$. Thus we can apply Theorem 8 to obtain a maximal modular right congruence $\delta$ for which $(e, g, v)$ is not congruent to $(u, w, v)$. Next consider the pair $(e, g, v)$ and $(e, p, v)$ where $g \neq p$. For any $l$ $\in L$ define $\alpha(k)=\phi(l, k)^{-1}$. Since $\alpha(e)^{-1} g p^{-1} \alpha(e) \neq 1$ there is a subgroup $H$ in $\mathscr{M}$ such that $\alpha(e)^{-1} g p^{-1} \alpha(e) \notin H$. The corresponding $\delta$ determined by Theorem 9 then separates $(e, g, v)$ and $(e, p, v)$.

THeOREM 11. Let $S$ be a Rees matrix semigroup with zero $\theta$. Then $S$ is semisimple if and only if $L_{0}$ is empty, $G=\{1\}, l(K)=l^{\prime}(K)$ implies $l=l^{\prime}$, and for every $k, k^{\prime}$ in $K$ there is an $f \in L$ such that $f(K)$ contains exactly one of $k$, $k^{\prime}$.

Proof. By Theorem 9 no maximal modular right congruence separ- 
ates $(e, g, f)$ and $(e, w, f)$ and none separates $(e, g, f)$ and $\theta$ for $f \in L_{0}$. Thus $G=\{1\}$ and $L_{0}$ is empty. If $l(K)=l^{\prime}(K)$ then $L(l)=L\left(l^{\prime}\right)$ and $(e, 1, l)$ and $\left(e, 1, l^{\prime}\right)$ are not separated. Hence we must have $l=l^{\prime}$. Finally if $e \neq u$ and $(e, 1, f)$ and $(u, 1, f)$ are to be separated there must be a $v$ in $L$ by Theorem 3 such that exactly one of $\phi(v, e)$ and $\phi(v, u)$ is nonzero.

Conversely, if $e \neq u$ there is a $v \in L$ such that exactly one of $\phi(v, e)$ and $\phi(v, u)$ is not zero. Say $\phi(v, e) \neq 0$. Let $K_{0}=\{k ; k \in K$ and $\phi(v, k)=0\}$ and $K_{1}=K-K_{0}$. The maximal modular right congruence related to this decomposition of $K$ separates $(e, 1, f)$ and $(u, 1, w)$. Finally since $L(f)=$ $L(w)$ implies $f=w$ we have $(e, 1, f)$ and $(e, 1, w)$ separated by any maximal modular right congruence if $f \neq w$.

A second definition of a radical [3] is the intersection of all the transitive or $\theta$-transitive right congruences on $S$. If this intersection is the identity relation we say $S$ is semisimple. In the subsequent theorems, as in Theorems 10 and 11, the use of the word semisimple applies to the immediately preceding definition of semisimplicity.

TheOREM 12. All Rees matrix semigroups $K \times G \times L$ without zero are semisimple if and only if either $G \neq\{1\}$ or $G=\{1\}$ and $K=\{e\}$.

Proof. The only condition on a right congruence to be transitive is that the decomposition of $K$ be trivial. Thus the right congruence defined by $H=\{1\}, \alpha(e)=1$ for all $e$ and $L(a, f)=\{f\}$ for all $a$ and $f$ is transitive. This right congruence separates all pairs $(e, g, f)$ and $(u, w, v)$ for which either $f \neq v$ or $g \neq w$. If $G \neq\{1\}$ we can modify our definition of $\delta$ slightly by choosing $\alpha(e)$ and $\alpha(u)$ in $G$ such that $\alpha(e)^{-1} \alpha(u) \neq 1$. Then under this definition the pair $(e, g, f)$ and $(u, g, f)$ are separated if $e \neq u$. If $G=\{1\}$ and $K=\{e\}$ all distinct pairs are separated by our first choice of $\delta$. Hence in either case $S$ is semisimple.

Conversely, assume $S$ is semisimple and $G=\{1\}$. Then $\alpha(k)=1$ for all $k$ and $(e, 1, f)$ is congruent to $(u, 1, f)$ for any transitive right congruence. Hence we must have $e=u$ and $K=\{e\}$.

THEOREM 13. A Rees matrix semigroup with zero is semisimple if and only if $L_{0}$ is empty.

Proof. If $L_{0}$ is empty let $\delta$ be the $\theta$-transitive right congruence defined by $H=\{1\}, \alpha \equiv 1, K_{1}=\{e\}, K_{0}=K-K_{1}$, and $L(a, f)=f$ for all $a \in G$ and $f \in L$. Then $\delta$ separates $(e, g, f)$ and $(u, w, v)$ if they are distinct.

Conversely, since for any $\theta$-transitive right congruence we have $\left(K_{0} \times\right.$ $\left.G \times L_{1}\right) \cup\left(K \times G \times L_{0}\right) \cup\{\theta\}$ in a single equivalence class we cannot 
separate any elements of $K \times G \times L_{0}$ from $\theta$. Hence we must have $L_{0}$ empty.

While the next two definitions of radicals are ideals, these elements still relate to right congruences. W. Jones $[4,6]$ defined an element of a semigroup to be right quasiregular if it is not a left identity for any modular right congruence except the universal right congruence. The radical was defined to be the largest ideal all of whose elements are right quasiregular. The semigroup $S$ was said to be semisimple in case the radical is empty or consists only of a zero element.

THEOREM 14. Any Rees matrix semigroup without zero is semisimple unless $G=\{1\}, L=\{f\}$ and $K$ is not a singleton.

Proof. If $L$ contains more than one element it has a decomposition $L$ $=L^{\prime} \cup L^{\prime \prime}$. The two subsets $K \times G \times L^{\prime}$ and $K \times G \times L^{\prime \prime}$ form a decomposition of $S$ related to a right congruence in which every element is a left identity. Let $H=\{1\}, \alpha(e)=\phi(f, e)^{-1}$ for all $e$. If $G \neq\{1\}$ the associated modular right congruence $\delta$ has $\left(e, \phi(f, e)^{-1}, f\right)$ as a left identity and $\delta \neq v$. If $L=\{f\}$ and $G=\{1\}$ the only modular right congruence is the universal relation. Hence to be semisimple the semigroup must be a singleton and the radical consists only of this zero element.

THEOREM 15. A Rees matrix semigroup with zero is semisimple if and only if $L_{0}$ is empty and for every $k \in K$ there is an $f \in L$ with $\phi(f, k) \neq 0$ (i.e., the semigroup is completely 0 -simple [1]).

Proof. If $S$ is semisimple then its radical consists only of $\theta$. If $\bar{K}=\{k$ $: \phi(f, k)=0$ for all $f \in L\}$ then by condition (b) of Theorem 3 it is clear that $\left(\bar{K} \times G \times L_{1}\right) \cup\left(K \times G \times L_{0}\right) \cup\{\theta\}$ is a two-sided ideal consisting of right quasiregular ideals. Hence both $\bar{K}$ and $L_{0}$ are empty.

Conversely, for any $e \in K$ let $f \in L$ such that $\phi(f, e) \neq 0$. Let $H=G$, $\alpha(k)=1$ for all $k, L(1, l)=\{l\}, K_{1}=\{k: \phi(f, k) \neq 0\}$ and $K_{0}=\{k: \phi(f$, $k)=0\}$. The associated modular right congruence has $\left(e, \phi(f, e)^{-1}, f\right)$ as a left identity. Hence $\left(e, \phi(f, e)^{-1}, f\right)$ is not right quasiregular. Since $e \times G \times$ $L$ is a minimal right ideal, none of its elements can be in the radical and $S$ is semisimple.

R. Slover [7] defined a right quasiregular element of a semigroup $S$ to be an element which is not a left identity for any right congruence which has one of its equivalence classes a right ideal, except the universal congruence. The radical is the maximal ideal all of whose elements are right 
quasiregular. A semigroup is semisimple if this ideal is empty or only the zero element.

THEOREM 16. A Rees matrix semigroup without zero is semisimple if and only if it is a singleton.

Proof. Clearly, if $S$ is a singleton it is semisimple.

Conversely, since there are no zero-transitive right congruences for $S$ every element is right quasiregular. Hence if $S$ is semisimple it must consist of only a zero element.

THEOREM 17. A Rees matrix semigroup with zero is semisimple if and only if $L_{0}$ is empty and for every $k \in K$ there is an $f \in L$ with $\phi(f, k) \neq 0$ (i.e., the semigroup is completely 0 -simple).

Proof. If $S$ has a zero element then the Jones radical equals the Slover radical [6]. Hence the result follows from Theorem 16.

Several other radicals are defined strictly in terms of ideals. The nilradical is the maximal ideal $N(S)$ such that for $X \in N(S)$ there is a power of $x$ that is a left zero. If this ideal is empty or $\{\theta\}$ the semigroup is said to be semisimple.

THEOREM 18. A Rees matrix semigroup $S$ without zero is semisimple unless $G=\{1\}, L=\{f\}$ and $K$ is not a singleton.

Proof. $S$ has no left zeros unless $G=\{1\}$ and $L=\{f\}$. In that case every element of $S$ is a left zero.

THEOREM 19. A Rees matrix semigroup $S$ with zero is semisimple if and only if $L_{0}$ is empty.

Proof. The set $\left(K \times G \times L_{0}\right) \cup\{\theta\}$ is an ideal of left zeros. Hence if $S$ is semisimple we have $L_{0}$ empty. Since $\left(K \times G \times L_{0}\right) \cup\{\theta\}$ includes all elements having some power a left zero, the converse is also true.

Another definition of semisimplicity is given [1] in terms of principal factors. It is then shown that a Rees matrix semigroup is semisimple if and only if $L_{0}$ is empty and $\{k: \phi(l, k)=0 \quad l \in L\}$ is empty.

Recently, still another definition of semisimplicity was given which appears to lead to a very successful structure theory [2]. Here the radical is 
defined in terms of three different types of ideals; each reflecting some degenerate behavior of the semigroup. We merely state here that under the corresponding definition of semisimplicity it is seen that all Rees matrix semigroups such that $L_{0}$ is empty are semisimple.

If we label different definitions of semisimplicity in order of introduction in this paper; $A$ (max. mod. rt. cong.), $B$ (trans. rt. cong.), $C$ (Jones rt. quasi-regularity), $D$ (Slover rt. quasi-regularity), $E$ (nilradical), $F$ (principal factors), $G$ (Flach ideals; we have the following charts of implications.

Rees matrix semigroup without zero:

$$
D \Rightarrow A \Rightarrow B \Rightarrow C \Longleftrightarrow E \Rightarrow G \Longleftrightarrow F \text {. }
$$

Rees matrix semigroups with zero:

$$
A \Rightarrow C \Longleftrightarrow D \Longleftrightarrow F \Rightarrow B \Longleftrightarrow E \Longleftrightarrow G .
$$

\section{REFERENCES}

1. A. H. Clifford, and G. B. Preston, The algebraic theory of semigroups, vol. 1., Amer. Math. Soc., (1961), Providence, R. I.

2. D. M. Flach, Semigroups with finiteness conditions, J. Algebra, (to appear).

3. H. J. Hoehnke, Structure of semigroups, Canad. J. Math. 18(1966), 449-491.

4. W. P. Jones, Semigroups Satisfying Ring-like Conditions, Doctoral Dissertation, Univ. of Iowa, 1969.

5. R. H. Oehmke, On maximal congruences and finite semisimple semigroups, Trans. Amer. Math. Soc., 125(1966), 223-237.

6. __ Quasi-regularity in Semigroups, Séminaire Dubreil-Pisot, $23^{\mathrm{e}}$ annea, 1969/70.

7. R. E. Slover, Representations of a semigroup, Trans. Amer. Math. Soc., 120(1965), 417-427.

Received April 19, 1973 and in revised form August 13, 1974. This work was accomplished with the partial support of ONR Grant N00014-68-A-0500.

UNIVERSITY OF IOWA 


\section{PACIFIC JOURNAL OF MATHEMATICS}

\section{EDITORS}

RICHARD ARENS (Managing Editor)

University of California

Los Angeles, California, 90024

\section{R. A. BEAUMONT}

University of Washington

Seattle, Washington 98105

\section{J. DugunDJI}

Department of Mathematics University of Southern California Los Angeles, California 90007

D. Gillbarg AND J. MilgRAM

Stanford University Stanford, California 94305

\section{ASSOCIATE EDITORS}

E. F. BECKENBACH

B. H. NEUMANN

SUPPORTING

UNIVERSITY OF BRITISH COLUMBIA

UNIVERSITY OF CALIFORNIA

MONTANA STATE UNIVERSITY

UNIVERSITY OF NEVADA

NEW MEXICO STATE UNIVERSITY

OREGON STATE UNIVERSITY

UNIVERSITY OF OREGON OSAKA UNIVERSITY
F. WOLF

K. YosHIDA

INSTITUTIONS

UNIVERSITY OF SOUTHERN CALIFORNIA

STANFORD UNIVERSITY

UNIVERSITY OF TOKYO

UNIVERSITY OF UTAH

WASHINGTON STATE UNIVERSITY

UNIVERSITY OF WASHINGTON

AMERICAN MATHEMATICAL SOCIETY

NAVAL WEAPONS CENTER

The Supporting Institutions listed above contribute to the cost of publication of this Journal, but they are not owners or publishers and have no responsibility for its content or policies.

Mathematical papers intended for publication in the Pacific Journal of Mathematics should be in typed form or offset-reproduced, (not dittoed), double spaced with large margins. Underline Greek letters in red, German in green, and script in blue. The first paragraph or two must be capable of being used separately as a synopsis of the entire paper. Items of the bibliography should not be cited there unless absolutely necessary, in which case they must be identified by author and Journal, rather than by item number. Manuscripts, in duplicate if possible, may be sent to any one of the five editors. Please classify according to the scheme of Math. Rev. Index to Vol. ${ }^{39}$. All other communications to the editors should be addressed to the managing editor, or Elaine Barth, University of California, Los Angeles, California, 90024.

100 reprints are provided free for each article, only if page charges have been substantially paid. Additional copies may be obtained at cost in multiples of 50 .

The Pacific Journal of Mathematics is issued monthly as of January 1966. Regular subscription rate: $\$ 72.00$ a year (6 Vols., 12 issues). Special rate: $\$ 36.00$ a year to individual members of supporting institutions.

Subscriptions, orders for back numbers, and changes of address should be sent to Pacific Journal of Mathematics, 103 Highland Boulevard, Berkeley, California 90708.

\section{PUBLISHED BY PACIFIC JOURNAL OF MATHEMATICS, A NON-PROFIT CORPORATION}

Copyright (C) 1974 by Pacific Journal of Mathematics

Manufactured and first issued in the U.S.A. 


\section{Pacific Journal of Mathematics}

\section{Vol. 54, No. $2 \quad$ June, 1974}

John Edward Coury, Walsh series with coefficients tending monotonically to

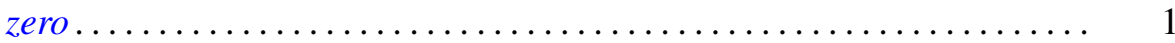

Patrick Michael Fitzpatrick and Walter Volodymyr Petryshyn, Fixed point theorems for multivalued noncompact acyclic mappings ............

Irving Leonard Glicksberg, More on Phragmén-Lindelöf for function

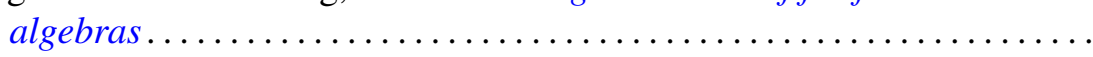

Adilson Goncalves, Structural constants. II .................. 39

Richard P. Gosselin, Closure theorems for affine transformation groups .... 53

Ralph Peter Grimaldi, Baer and UT-modules over domains ........... 59

Edward Grossman, On the prime ideal divisors of $\left(a^{n}-b^{n}\right) \ldots \ldots \ldots \ldots . \ldots 73$

A. Hedayat and Ester Seiden, On the theory and application of sum composition of Latin squares and orthogonal Latin squares.......... .

Gerald L. Itzkowitz, Continuous measures, Baire category, and uniform continuity in topological groups ......................... 115

Francis Masat, Right simple congruences on a semigroup ............ 127

Robert Harvey Oehmke, Right congruences and semisimplicity for Rees matrix semigroups..................................

Qazi Ibadur Rahman and Jan Stankiewicz, Differential inequalities and local valency . . . . . . . . . . . . . . . . . . . . . . . . . . . . . . . . . . . . . 165

William John Reed, Random points in a simplex ................ 183

Mohan S. Shrikhande, Strongly regular graphs and group divisible

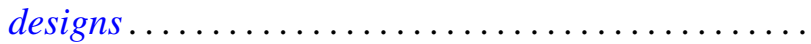

Zahava Shmuely, The structure of Galois connections ... . .

Robert C. Shock, Dual generalizations of the Artinian and Noetherian

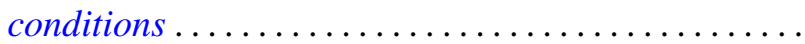

Arne Stray, Approximation and interpolation for some spaces of analytic functions in the unit disc ........................

Eldon Jon Vought, Monotone decompositions into trees of Hausdorff continua irreducible about a finite subset ............

James Wirth, The mapping cylinder axiom for WCHP fibrations ... 263

Gordon S. Woodward, Invariant means and ergodic sets in Fourier analysis... 